\title{
Perturbations of intermediate $\mathrm{C}^{*}$-subalgebras for simple $\mathrm{C}^{*}$-algebras
}

\author{
SHOJI INO AND YASUO WATATANI
}

\begin{abstract}
We study uniform perturbations of intermediate $\mathrm{C}^{*}$-subalgebras of inclusions of simple $\mathrm{C}^{*}$-algebras. If a unital simple $\mathrm{C}^{*}$-algebra has a simple $\mathrm{C}^{*}$-subalgebra of finite index, then sufficiently close simple intermediate $\mathrm{C}^{*}$-subalgebras are unitarily equivalent. These $\mathrm{C}^{*}$ subalgebras need not to be nuclear. The unitary can be chosen in the relative commutant algebra. An imediate corollary is the following: If the relative commutant is trivial, then the set of intermediate $\mathrm{C}^{*}$-subagebras is a finite set.
\end{abstract}

\section{INTRODUCTION}

The study of the uniform perturbation theory of operator algebras was started with 11 by Kadison and Kastler in 1972. They introduced a metirc on the set of $\mathrm{C}^{*}$-subalgebras of a fixed $\mathrm{C}^{*}$-algebra by the Hausdorff distance between the unit balls. A conjugacy by a unitary near to the identity gives close $\mathrm{C}^{*}$-subalgebras. They conjectured that suitably close $\mathrm{C}^{*}$-algebras must be unitarily equivalent. Although Choi and Christensen gave counterexamples to the conjecture in [2], the conjecture has been verified in various situations. The problem was solved positively when one algebra is separable and $\mathrm{AF}$ in Chiristensen [5] and separable $\mathrm{C}^{*}$-algebras of continuous trace by Phillips-Raeburn [16. Khoshkam [13] showed that sufficiently close nuclear $\mathrm{C}^{*}$-algebras have isomorphic K-groups. Recently Christensen, Sinclair, Smith, White and Winter have solved it completely when one algebra is separable and nuclear in [6].

In this paper we study uniform perturbations of intermediate $\mathrm{C}^{*}$-subalgebras of inclusions of simple $\mathrm{C}^{*}$-algebras. If a unital simple $\mathrm{C}^{*}$-algebra has a simple $\mathrm{C}^{*}$-subalgebra of finite index, then sufficiently close simple intermediate $\mathrm{C}^{*}$-subalgebras are unitarily equivalent. Thanks to Izumi's result in [9], we do not need to assume the existence of the conditional expectations onto intermediate $\mathrm{C}^{*}$-subalgebras apriori. Furthermore, if the relative commutant is trivial, then the set of intermediate $\mathrm{C}^{*}$-subagebras is a finite set.

In the case of subfactor theory of Jones [10], the lattices of intermediate subfactors with their finiteness were studied in Popa [17, Watatani [20], Teruya-Watatani [18, Longo [15], KhoshkamMashhood [14, Grossman-Jones [8, Grossman-Izumi [7] and Xu [21] for example. In these study, the $\|\cdot\|_{2}$-perterbation technique of von Neumann algebras developed by Christensen [4] are essentially used.

We prepare to consider uniform perturbations of intermediate $\mathrm{C}^{*}$-subalgebras of inclusions of not necessarily simple $\mathrm{C}^{*}$-algebras. But in general, we need to assume the existance of the conditional expectations onto intermediate $\mathrm{C}^{*}$-subalgebras to get a positive solution for the moment.

\section{MEtric AND INDEX FOR $\mathrm{C}^{*}$-SUbALGEBRAS}

Let $C$ be a $\mathrm{C}^{*}$-algebra. We denote by $C_{1}$ the unit ball of $C$. We recall a metric on the set of all subalgebras of a $\mathrm{C}^{*}$-algebra $C$ after Kadison and Kastler [11]. Let $A$ and $B$ be $\mathrm{C}^{*}$-subalgebras of $C$. Then the distance $d(A, B)$ between $A$ and $B$ is defined by the Hausdorff distance between 
the unit balls of $A$ and $B$, that is,

$$
d(A, B)=\max \left\{\sup _{a \in A_{1}} \inf _{b \in B_{1}}\|a-b\|, \sup _{b \in B_{1}} \inf _{a \in A_{1}}\|b-a\|\right\} .
$$

Therefore if $d(A, B)<\gamma$, then for each $x$ in the unit ball of either $A$ or $B$, there exists $y$ in the unit ball of the other algebra with $\|x-y\|<\gamma$.

We need the following known fact to show that a desired inclusion is onto.

Lemma 2.1. Let $A$ and $B$ be $\mathrm{C}^{*}$-subalgebras of $a \mathrm{C}^{*}$-algebra. If $A \subset B$ and $d(A, B)<1$, then $A=B$.

The next proposition records some standard estimates.

Lemma 2.2. Let $A$ be a unital $\mathrm{C}^{*}$-algebra.

(1) Let $x \in A$ satisfy that $\|x-1\|<1$ and $u \in A$ be the unitary in the polar decomposition $x=u|x|$. Then

$$
\|u-1\| \leq \sqrt{2}\|x-1\| .
$$

(2) Let $p \in A$ be a projection and $a \in A$ be self-adjoint. Assme that $\delta:=\|a-p\|<1 / 2$. Let $q=\chi_{[1-\delta, 1+\delta]}(a)$. Then $q$ is a projection in $C^{*}(a, I)$ such that $\|q-p\| \leq 2\|a-p\|<1$.

(3) Let $p$ and $q$ be projections in $A$ with $\|p-q\|<1$. Then there exists a unitary $w \in A$ such that

$$
w p w^{*}=q, \quad \text { and } \quad\|w-1\| \leq \sqrt{2}\|p-q\| .
$$

We recall some basic facts on index for $\mathrm{C}^{*}$-subalgebas in [19].

Definition 2.3. Let $B$ be a $C^{*}$-algebra and $A$ a $C^{*}$-subalgebra of $B$ with a common unit. Let $E$ be a conditional expectation of $B$ onto $A$. We say that $E$ is of finite index if there exists a finite set $\left\{u_{1}, \ldots, u_{N}\right\} \subset B$, called a (quasi-)basis for $E$, such that

$$
b=\sum_{i=1}^{N} u_{i} E\left(u_{i}^{*} b\right), \quad \text { for any } b \in B .
$$

When $E$ is of finite index, then the index of $E$ is defined by

$$
\text { Index } E=\sum_{i=1}^{N} u_{i} u_{i}^{*} \text {. }
$$

The value Index $E$ is in the center of $B$ and does not depend on the choice of a basis for $E$. Moreover Index $E$ is positive invertible operator in $B$. In fact Index $E \geq I$.

We can choose a basis for $E$ in the unit ball of $B$ if it is necessary. In fact, choose a positive integer $K$ such that $K \geq \max \left\{\left\|u_{1}\right\|, \ldots,\left\|u_{N}\right\|\right\}$. Define

$$
v_{(i-1) K+j}=\frac{1}{\sqrt{K}} u_{i}, \quad \text { for } i=1,2, \ldots, N, j=1,2, \ldots, K .
$$

Then $\left\{v_{1}, \ldots, v_{K N}\right\}$ is a desired basis.

Next, we recall the $\mathrm{C}^{*}$-basic construction. Let $E: B \rightarrow A$ be a faithful conditional expectation. Define a $A$-valued inner product on $B$ by

$$
\langle x, y\rangle_{A}=\underset{2}{E\left(x^{*} y\right)}, \quad x, y \in B .
$$


We denote by $\mathcal{E}$ the completion of $B$. Then $\mathcal{E}$ becomes a Hilbert $A$-module. Let $\eta: B \rightarrow \mathcal{E}$ be the natural inclusion map. Thus $\|\eta(x)\|=\left\|E\left(x^{*} x\right)\right\|^{1 / 2}$. Let $\mathcal{L}_{A}(\mathcal{E})$ be the set of bounded $A$-module maps on $\mathcal{E}$ with adjoints. Let $\mathcal{K}_{A}(\mathcal{E})$ be the set of "compact" operators on $\mathcal{E}$.

For $b \in B$, define $\lambda(b) \in \mathcal{L}_{A}(\mathcal{E})$ by

$$
\lambda(b) \eta(x)=\eta(b x), \quad x \in B .
$$

Then $\lambda: B \rightarrow \mathcal{L}_{A}(\mathcal{E})$ turns out to be an injective $*$-homomorphism. Define the Jones projection $e_{A} \in \mathcal{L}_{A}(\mathcal{E})$ by

$$
e_{A} \eta(x)=\eta(E(x)), \quad x \in B
$$

The $\mathrm{C}^{*}$-basic construction $C^{*}\left\langle B, e_{A}\right\rangle$ is defined by the closure of the linear span of $\left\{\lambda(x) e_{A} \lambda(y) \mid x, y \in\right.$ $B\}$.

Moreover $\lambda$ and $e_{A}$ satisfy the following:

(1) (covariant relation) $e_{A} \lambda(b) e_{A}=\lambda(E(b)) e_{A}$ for $b \in B$.

(2) Let $b \in B$. Then $b$ is in $A$ if and only if $e_{A} \lambda(b)=\lambda(b) e_{A}$.

Definition 2.4. Let $D$ be a $\mathrm{C}^{*}$-algebra and $C$ a $\mathrm{C}^{*}$-subalgebra of $D$ with a common unit. Assume that the inclusion $C \subset D$ has a conditional expectation $E_{C}^{D}: D \rightarrow C$ of finte index. We denote by $\operatorname{IMS}\left(C, D, E_{C}^{D}\right)$ the set of all intermediate $\mathrm{C}^{*}$-subalgebra $A$ between $C$ and $D$ with a conditional expectation $E_{A}^{D}: D \rightarrow A$ satisfying the compatibility condition $E_{C}^{A} \circ E_{A}^{D}=E_{C}^{D}$, where $E_{C}^{A}:=\left.E_{C}^{D}\right|_{A}: A \rightarrow C$ is the coditional expectation defined by the restriction of $E_{C}^{D}$ to $A$.

Let $A$ be in $\operatorname{IMS}\left(C, D, E_{C}^{D}\right)$. If there exists another conditional expectation $F_{A}^{D}: D \rightarrow A$ satisfying the compatibility condition $E_{C}^{A} \circ F_{A}^{D}=E_{C}^{D}$, then $F_{A}^{D}=E_{A}^{D}$. In fact for any $x \in D$ and $a \in A$, we have that $E_{C}^{D}\left(F_{A}^{D}(a x)\right)=E_{C}^{D}(a x)$. Hence

$$
E_{C}^{D}\left(a F_{A}^{D}(x)\right)=E_{C}^{D}(a x)=E_{C}^{D}\left(a E_{A}^{D}(x)\right) .
$$

Then $E_{C}^{D}\left(a\left(F_{A}^{D}(x)-E_{A}^{D}(x)\right)\right)=0$. Put $a=\left(F_{A}^{D}(x)-E_{A}^{D}(x)\right)^{*}$. Since $E_{C}^{D}$ is of finite index, $E_{C}^{D}$ is faithful. This implies that $F_{A}^{D}=E_{A}^{D}$.

For any $A$ in $\operatorname{IMS}\left(C, D, E_{C}^{D}\right)$, the conditional expectation $E_{C}^{A}=\left.E_{C}^{D}\right|_{A}: A \rightarrow C$ is of finite index. In fact, let $\left\{u_{1}, \ldots, u_{N}\right\} \subset D$ be a basis of $E_{C}^{D}$. Put $v_{i}=E_{A}^{D}\left(u_{i}\right)$. Then $\left\{v_{1}, \ldots, v_{N}\right\} \subset D$ is a basis of $E_{C}^{A}$. If $u_{i}$ is in $D_{1}$, then $v_{i}$ is in $A_{1}$.

But we should be careful that an intermediate $\mathrm{C}^{*}$-subalgebra $A$ between $C$ and $D$ may not have a conditional expectation $E_{A}^{D}: D \rightarrow A$ in general.

Example 2.5. Let $D=C\left([0,1], M_{2}(\mathbb{C})\right)$ be the algebra of $2 \times 2$ matrix valued continuous functions on the unit interval $[0,1]$. Consider a $\mathrm{C}^{*}$-subalgebra $C=C([0,1], \mathbb{C} I) \subset D$. Then there exist a conditional expectation $E_{C}^{D}: D \rightarrow C$ of finte index. In fact, define $\left(E_{C}^{D}(f)\right)(x)=$ $\operatorname{tr}(f(x)) I$ for $f \in D$ and $x \in[0,1]$, where $\operatorname{tr}$ is the normalized trace on $M_{2}(\mathbb{C})$. Define an intermediate $\mathrm{C}^{*}$-subalgebra $A$ between $C$ and $D$ by

$$
A:=\left\{f \in C\left([0,1], M_{2}(\mathbb{C})\right) \mid f\left(\frac{1}{2}\right) \in \mathbb{C} I\right\}
$$

Then there exist no conditional expectation of $D$ onto $A$. In fact, suppose that there were a conditional expectation $E_{A}^{D}: D \rightarrow A$. We can choose $a \in C([0,1], \mathbb{C})$ such that $a(1 / 2)=0$ and 
$a(x) \neq 0$ for any $x \neq 1 / 2$. Since $\left(\begin{array}{cc}a & 0 \\ 0 & 0\end{array}\right),\left(\begin{array}{ll}a & 0 \\ 0 & a\end{array}\right) \in A$, we have

$$
\left(\begin{array}{ll}
a & 0 \\
0 & 0
\end{array}\right)=E_{A}^{D}\left(\left(\begin{array}{ll}
a & 0 \\
0 & 0
\end{array}\right)\right)=E_{A}^{D}\left(\left(\begin{array}{ll}
1 & 0 \\
0 & 0
\end{array}\right)\left(\begin{array}{ll}
a & 0 \\
0 & a
\end{array}\right)\right)=E_{A}^{D}\left(\left(\begin{array}{ll}
\mathbf{1} & 0 \\
0 & 0
\end{array}\right)\right)\left(\begin{array}{ll}
a & 0 \\
0 & a
\end{array}\right),
$$

where $\mathbf{1}$ is an identity of $C([0,1], \mathbb{C})$. This shows that $E_{A}^{D}\left(\left(\begin{array}{ll}\mathbf{1} & 0 \\ 0 & 0\end{array}\right)\right)(x)=\left(\begin{array}{ll}1 & 0 \\ 0 & 0\end{array}\right)$ for any $x \neq$ 1/2. By the continuity, $E_{A}^{D}\left(\left(\begin{array}{ll}\mathbf{1} & 0 \\ 0 & 0\end{array}\right)\right)=\left(\begin{array}{ll}\mathbf{1} & 0 \\ 0 & 0\end{array}\right)$. But $\left(\begin{array}{ll}\mathbf{1} & 0 \\ 0 & 0\end{array}\right) \notin A$. This is a contradiction.

There is a relation between $d(A, B)$ and the norm estimate $\left\|e_{A}-e_{B}\right\|$ of Jones projections $e_{A}$ and $e_{B}$ for intermediate $\mathrm{C}^{*}$-subalgebras $A, B \in \operatorname{IMS}\left(C, D, E_{C}^{D}\right)$. More precisely, let $\mathcal{E}$ be the completion of $D$ by the $C$-valued inner procuct $E_{C}^{D}\left(x^{*} y\right)$. Then $\mathcal{E}$ becomes a Hilbert $C$-module. Let $\eta: D \rightarrow \mathcal{E}$ be the natural inclusion map. We can define Jones projections for intermediate $C^{*}$-subalgebras by $e_{A} \eta(x)=\eta\left(E_{A}^{D}(x)\right)$ and $e_{B} \eta(x)=\eta\left(E_{B}^{D}(x)\right)$ for $x \in D$. These projections also enjoy similar properties with a usual Jones projection $e_{C}$. For example, $e_{A}$ commute with the left multiplication operator $\lambda(a)$ for $a \in A$.

Lemma 2.6. Let $D$ be a $\mathrm{C}^{*}$-algebra and $C$ a $\mathrm{C}^{*}$-subalgebra of $D$ with a common unit and $E_{C}^{D}: D \rightarrow C$ a conditional expectation of finite index. Then for $A, B \in \operatorname{IMS}\left(C, D, E_{C}^{D}\right)$ we have that

$$
d(A, B) \leq\left\|\operatorname{Index} E_{C}^{D}\right\|\left\|e_{A}-e_{B}\right\| .
$$

Proof. Put $c=\left\|\operatorname{Index} E_{C}^{D}\right\|$. Then for any $a \in A_{1}$, we have $\|\eta(a)\| \leq\|a\| \leq 1$. By the PimsnerPopa inequality, $E\left(x^{*} x\right) \geq c^{-1} x^{*} x$ of Proposition 2.6.2 in [19],

$$
\begin{aligned}
\left\|e_{A}-e_{B}\right\| & \geq \frac{\left\|\eta\left(E_{A}^{D}(a)-E_{B}^{D}(a)\right)\right\|}{\|\eta(a)\|} \geq\left\|\eta\left(a-E_{B}^{D}(a)\right)\right\| \\
& =\left\|E_{C}^{D}\left(\left(a-E_{B}^{D}(a)\right)^{*}\left(a-E_{B}^{D}(a)\right)\right)\right\|^{1 / 2} \\
& \geq \frac{1}{c}\left\|\left(a-E_{B}^{D}(a)\right)^{*}\left(a-E_{B}^{D}(a)\right)\right\|^{1 / 2}=\frac{1}{c}\left\|a-E_{B}^{D}(a)\right\| .
\end{aligned}
$$

Therefore for any $a \in A_{1}$, we can find $b:=E_{B}^{D}(a) \in B_{1}$ such that $\|a-b\| \leq c\left\|e_{A}-e_{B}\right\|$. By a symmmetric argument, we have that $d(A, B) \leq c\left\|e_{A}-e_{B}\right\|$.

\section{Perturbations}

We begin with some elementary estimations.

Lemma 3.1. Let $A$ and $D$ be $\mathrm{C}^{*}$-algebras. Let $\varphi: A \rightarrow D$ be a contractive positive map and $\psi: A \rightarrow D$ be $a *$-homomorphism. Then for any $x, y \in A$

$$
\|\varphi(x y)-\varphi(x) \varphi(y)\| \leq 3\|\varphi-\psi\|\|x\|\|y\| .
$$

Proof. Approximate $\varphi$ by $\psi$.

Lemma 3.2. Let $D$ be a $\mathrm{C}^{*}$-algebra and $A, B$ be $\mathrm{C}^{*}$-subalgebras of $D$. Let $E_{B}: D \rightarrow B$ be a conditional expectation. Consider the restriction map $\left.E_{B}\right|_{A}: A \rightarrow D$ and an inclusion map $\iota_{A}: A \rightarrow D$. Then we have

$$
\left\|\left.E_{B}\right|_{A}-\iota_{A}\right\| \leq 2 d(A, B)
$$


and for any $x, y \in A$,

$$
\left\|E_{B}(x y)-E_{B}(x) E_{B}(y)\right\| \leq 6 d(A, B)\|x\|\|y\| .
$$

Proof. For any $\epsilon>0$ and $a \in A_{1}$, there exists $b \in B_{1}$ such that $\|a-b\| \leq d(A, B)+\varepsilon / 2$. Then

$$
\left\|E_{B}(a)-\iota_{A}(a)\right\| \leq\left\|E_{B}(a-b)\right\|+\|a-b\| \leq 2 d(A, B)+\varepsilon .
$$

Hence $\left\|\left.E_{B}\right|_{A}-\iota_{A}\right\| \leq 2 d(A, B)$. The rest is clear.

We shall show that two close intermediate $\mathrm{C}^{*}$-subalgebras $A, B \in \operatorname{IMS}\left(C, D, E_{C}^{D}\right)$ are unitarily equivalent. We need the following two key lemmas:

Lemma 3.3. Let $D$ be a $\mathrm{C}^{*}$-algebra and $C$ a $\mathrm{C}^{*}$-subalgebra of $D$ with a common unit. Let $E_{C}^{D}: D \rightarrow C$ be a conditional expectation of finite index with a basis $\left\{u_{1}, \ldots, u_{N}\right\}$ in $D_{1}$. For any $A, B \in \operatorname{IMS}\left(C, D, E_{C}^{D}\right)$, if $d(A, B)<\left(24 N^{2}\right)^{-1}$, then there exists a unital $*$-homomorphism $\psi: A \rightarrow B$ such that $\left.\psi\right|_{C}=i d_{C}$ and

$$
\left\|\left.E_{B}^{D}\right|_{A}-\psi\right\| \leq 8 \sqrt{3} N \sqrt{d(A, B)} .
$$

Proof. Let $\mathcal{E}$ be the Hilbert $B$-module completion of $D$ using $E_{B}^{D}$ and $\eta$ the natural inclusion map from $D$ to $\mathcal{E}$. Define an injective $*$-homomorphism $\lambda: D \rightarrow \mathcal{L}_{C}(\mathcal{E})$ by

$$
\lambda(d) \eta(x)=\eta(d x), \quad d, x \in D .
$$

Then for any $b \in B$, we have $\lambda(b) e_{B}=e_{B} \lambda(b)$. The map $B \ni b \mapsto \lambda(b) e_{B} \in \mathcal{L}_{C}(\mathcal{E})$ is a injective $*$-homomorphism and $\left\|\lambda(b) e_{B}\right\|=\|\lambda(b)\|$. For any $z \in D$, we have $e_{B} \lambda(z) e_{B}=E_{B}^{D}(z) e_{B}$. Hence for any $x, y \in D$,

$$
\begin{aligned}
& \left\|E_{B}^{D}(x y)-E_{B}^{D}(x) E_{B}^{D}(y)\right\|=\left\|\lambda\left(E_{B}^{D}(x y)-E_{B}^{D}(x) E_{B}^{D}(y)\right) e_{B}\right\| \\
& =\left\|\lambda\left(E_{B}^{D}(x y)\right) e_{B}-\lambda\left(E_{B}^{D}(x)\right) e_{B} \lambda\left(E_{B}^{D}(y)\right) e_{B}\right\| \\
& =\left\|e_{B} \lambda(x y) e_{B}-e_{B} \lambda(x) e_{B} \lambda(y) e_{B}\right\|=\left\|e_{B} \lambda(x)\left(I-e_{B}\right) \lambda(y) e_{B}\right\| .
\end{aligned}
$$

Let $E_{C}^{A}$ be a restriction of $E_{C}^{D}$ to $A$ and put $v_{i}=E_{A}^{D}\left(u_{i}\right)$. Then $\left\{v_{1}, \ldots, v_{N}\right\}$ is a basis for $E_{C}^{A}$ in $A_{1}$. Define an operator

$$
t=\sum_{i=1}^{N} \lambda\left(\left(\operatorname{Index} E_{C}^{A}\right)^{-1}\right) \lambda\left(v_{i}\right) e_{B} \lambda\left(v_{i}^{*}\right) \in \mathcal{L}_{C}(\mathcal{E}) .
$$

Recall that Index $E_{C}^{A}$ is in the center of $A$. For any $a \in A$

$$
\begin{aligned}
\lambda(a) t & =\sum_{i=1}^{N} \lambda\left(\left(\operatorname{Index} E_{C}^{A}\right)^{-1}\right) \lambda\left(a v_{i}\right) e_{B} \lambda\left(v_{i}^{*}\right) \\
& =\sum_{i=1}^{N} \sum_{j=1}^{N} \lambda\left(\left(\operatorname{Index} E_{C}^{A}\right)^{-1}\right) \lambda\left(v_{j} E_{C}^{A}\left(v_{j}^{*} a v_{i}\right)\right) e_{B} \lambda\left(v_{i}^{*}\right) \\
& =\sum_{j=1}^{N} \lambda\left(\left(\operatorname{Index} E_{C}^{A}\right)^{-1}\right) \lambda\left(v_{j}\right) e_{B} \sum_{i=1}^{N} \lambda\left(E_{C}^{A}\left(v_{j}^{*} a v_{i}\right) v_{i}^{*}\right) \\
& =\sum_{j=1}^{N} \lambda\left(\left(\operatorname{Index} E_{C}^{A}\right)^{-1}\right) \lambda\left(v_{j}\right) e_{B} \lambda\left(v_{j}^{*}\right) \lambda(a)=t \lambda(a) .
\end{aligned}
$$


Since Index $E_{C}^{A}=\sum_{i} v_{i} v_{i}^{*}$ and Lemma $\underline{3.2}$,

$$
\begin{aligned}
\left\|t-e_{B}\right\| & =\left\|t-\sum_{i=1}^{N} \lambda\left(\left(\operatorname{Index} E_{C}^{A}\right)^{-1}\right) \lambda\left(v_{i}\right) \lambda\left(v_{i}^{*}\right) e_{B}\right\| \\
& \leq \sum_{i=1}^{N}\left\|\lambda\left(\left(\operatorname{Index} E_{C}^{A}\right)^{-1}\right)\right\|\left\|\lambda\left(v_{i}\right)\right\|\left\|e_{B} \lambda\left(v_{i}^{*}\right)-\lambda\left(v_{i}^{*}\right) e_{B}\right\| \\
& \leq N\left\|e_{B} \lambda\left(v_{i}^{*}\right)-\lambda\left(v_{i}^{*}\right) e_{B}\right\| \\
& =N\left\|\left(I-e_{B}\right) \lambda\left(v_{i}^{*}\right) e_{B}-e_{B} \lambda\left(v_{i}^{*}\right)\left(I-e_{B}\right)\right\| \\
& =N \cdot \max \left\{\left\|\left(I-e_{B}\right) \lambda\left(v_{i}^{*}\right) e_{B}\right\|,\left\|e_{B} \lambda\left(v_{i}^{*}\right)\left(I-e_{B}\right)\right\|\right\} \\
& =N \cdot \max \left\{\left\|e_{B} \lambda\left(v_{i}\right)\left(I-e_{B}\right) \lambda\left(v_{i}^{*}\right) e_{B}\right\|^{1 / 2},\left\|e_{B} \lambda\left(v_{i}^{*}\right)\left(I-e_{B}\right) \lambda\left(v_{i}\right) e_{B}\right\|^{1 / 2}\right\} \\
& =N \cdot \max \left\{\left\|E_{B}^{D}\left(v_{i} v_{i}^{*}\right)-E_{B}^{D}\left(v_{i}\right) E_{B}^{D}\left(v_{i}^{*}\right)\right\|^{1 / 2},\left\|E_{B}^{D}\left(v_{i}^{*} v_{i}\right)-E_{B}^{D}\left(v_{i}^{*}\right) E_{B}^{D}\left(v_{i}\right)\right\|^{1 / 2}\right\} \\
& \leq N \sqrt{6 d(A, B)}<\frac{1}{2} .
\end{aligned}
$$

Put $\delta=\left\|t-e_{B}\right\|=\left\|\left(t+t^{*}\right) / 2-e_{B}\right\|$. Let $q=\chi_{[1-\delta, 1+\delta]}\left(\left(t+t^{*}\right) / 2\right)$. By Lemma 2.2, $q$ is a projection in $C^{*}\left(t, I_{\mathcal{E}}\right)$ and commutes with $\lambda(a)$ for any $a \in A$, and $\left\|q-e_{B}\right\| \leq 2\left\|t-e_{B}\right\|<1$. Therefore there exists a unitary $w \in C^{*}\left(t, e_{B}, I_{\mathcal{E}}\right) \subset \mathcal{L}_{B}(\mathcal{E})$ such that $w e_{B} w^{*}=q$ and $\left\|w-1_{\mathcal{E}}\right\| \leq$ $\sqrt{2}\left\|q-e_{B}\right\|$. Define $\psi^{\prime}: A \rightarrow \mathcal{L}_{B}(\mathcal{E})$ by

$$
\psi^{\prime}(a)=w^{*} q \lambda(a) q w=e_{B} w^{*} \lambda(a) w e_{B}, \quad a \in A .
$$

Since the projection $q$ commutes with $\lambda(a)$, it is clear that $\psi^{\prime}$ is a $*$-homomorphism.

The unitary $w$ is in $C^{*}\left(t, e_{B}, I_{\mathcal{E}}\right) \subset C^{*}\left(\lambda(A), e_{B}, I_{\mathcal{E}}\right)$, and $e_{B} w^{*} \lambda(a) w e_{B} \in \lambda(B) e_{B}$ for $a \in A$. Therefore, $\psi^{\prime}(A) \subset \lambda(B) e_{B} \subset \mathcal{L}_{B}(\mathcal{E})$. Let $\mathcal{E}^{\prime}$ be a closure of $\eta(B)$ in $\mathcal{E}$. Define an injective *-homomorphism $\lambda^{\prime}: B \rightarrow \mathcal{L}_{B}\left(\mathcal{E}^{\prime}\right)$ by

$$
\lambda^{\prime}(b) \eta(x)=\eta(b x), \quad b, x \in B,
$$

and a surjective $*$-isomorphism $\iota: \lambda(B) e_{B} \rightarrow \lambda^{\prime}(B)$ by

$$
\iota\left(\lambda(b) e_{B}\right)=\lambda^{\prime}(b), \quad b \in B
$$

Then $\lambda(B) e_{B}$ is isomorphic to $\lambda^{\prime}(B)$. Thus, we can define a $*$-homomorphism $\psi=\left(\lambda^{\prime}\right)^{-1} \circ \iota \circ \psi^{\prime}$ : $A \rightarrow B$, that is, $\lambda(\psi(a)) e_{B}=\psi^{\prime}(a)$. Then for any contraction $a \in A$,

$$
\begin{aligned}
\left\|\left.E_{B}^{D}\right|_{A}(a)-\psi(a)\right\| & =\left\|e_{B}\left(\lambda\left(\left.E_{B}^{D}\right|_{A}(a)-\psi(a)\right)\right) e_{B}\right\|=\left\|e_{B} \lambda\left(E_{B}^{D}(a)\right) e_{B}-\psi^{\prime}(a)\right\| \\
& =\left\|e_{B} \lambda(a) e_{B}-e_{B} w^{*} \lambda(a) w e_{B}\right\| \\
& =\left\|e_{B} \lambda(a)\left(I-w^{*}\right) e_{B}+e_{B}\left(I-w^{*}\right) \lambda(a) w e_{B}\right\| \leq 2\|w-I\| .
\end{aligned}
$$

Therefore

$$
\left\|\left.E_{B}^{D}\right|_{A}-\psi\right\| \leq 2\|w-I\| \leq 8 \sqrt{3} N \sqrt{d(A, B)}
$$

Since $w$ is contained in $C^{*}\left(t, e_{B}, I_{\mathcal{E}}\right), C \subset A$ and $C \subset B$, we have that $\lambda(c) w=w \lambda(c)$ for $c \in C$. Hence

$$
\psi^{\prime}(c)=e_{B} w^{*} \lambda(c) w e_{B}=e_{B} \lambda(c) e_{B}=\lambda(c) e_{B}
$$

Therefore $\psi(c)=c=i d_{C}(c)$ for any $c \in C$.

Next Lemma shows how to find an intertwiner for close $*$-homomorphisms. 
Lemma 3.4. Let $D$ be a $\mathrm{C}^{*}$-algebra and $C$ a $\mathrm{C}^{*}$-subalgebra of $D$ with a common unit and $E_{C}^{D}: D \rightarrow C$ be a conditional expectation of finite index with a basis $\left\{u_{1}, \ldots, u_{N}\right\}$ in $D_{1}$. For any $A \in \operatorname{IMS}\left(C, D, E_{C}^{D}\right)$, If $\phi_{1}, \phi_{2}: A \rightarrow D$ are unital $*$-homomorphisms such that $\left.\phi_{1}\right|_{C}=i d_{C}=$ $\left.\phi_{2}\right|_{C}$ and $\left\|\phi_{1}-\phi_{2}\right\|<1 / N$, then there exists a unitary $u \in D$ such that $\phi_{1}=\operatorname{Ad}(u) \circ \phi_{2}$ and $\left\|u-I_{D}\right\|<\sqrt{2} N\left\|\phi_{1}-\phi_{2}\right\|$.

Proof. Let $E_{C}^{A}$ be the estriction of $E_{C}^{D}$ to $A$ and $v_{i}=E_{A}^{D}\left(u_{i}\right)$. Put

$$
s=\sum_{i=1}^{N} \phi_{1}\left(\left(\operatorname{Index} E_{C}^{A}\right)^{-1}\right) \phi_{1}\left(v_{i}\right) \phi_{2}\left(v_{i}^{*}\right) .
$$

Since $\left\|\left(\operatorname{Index} E_{C}^{A}\right)^{-1}\right\| \leq 1$,

$$
\begin{aligned}
\left\|s-I_{D}\right\| & =\left\|s-\sum_{i=1}^{N} \phi_{1}\left(\left(\operatorname{Index} E_{C}^{A}\right)^{-1}\right) \phi_{1}\left(v_{i}\right) \phi_{1}\left(v_{i}^{*}\right)\right\| \\
& \leq \sum_{i=1}^{N}\left\|\phi_{1}\left(\left(\operatorname{Index} E_{C}^{A}\right)^{-1}\right) \phi_{1}\left(v_{i}\right)\right\|\left\|\phi_{2}\left(v_{i}^{*}\right)-\phi_{1}\left(v_{i}^{*}\right)\right\| \\
& \leq N\left\|\phi_{1}-\phi_{2}\right\|<1 .
\end{aligned}
$$

Therefore the unitary $u$ in the polar decomposition $s=u|s|$ lies in $D$ and satisfies $\left\|u-I_{D}\right\| \leq$ $\sqrt{2} N\left\|\phi_{1}-\phi_{2}\right\|$ by Lemma 2.2. Furthermore, for any $a \in A$,

$$
\begin{aligned}
\phi_{1}(a) s & =\sum_{i=1}^{N} \phi_{1}\left(\left(\operatorname{Index} E_{C}^{A}\right)^{-1}\right) \phi_{1}\left(a v_{i}\right) \phi_{2}\left(v_{i}^{*}\right) \\
& =\sum_{i=1}^{N} \sum_{j=1}^{N} \phi_{1}\left(\left(\operatorname{Index} E_{C}^{A}\right)^{-1}\right) \phi_{1}\left(v_{j} E_{C}^{A}\left(v_{j}^{*} a v_{i}\right)\right) \phi_{2}\left(v_{i}^{*}\right) \\
& =\sum_{i=1}^{N} \sum_{j=1}^{N} \phi_{1}\left(\left(\operatorname{Index} E_{C}^{A}\right)^{-1}\right) \phi_{1}\left(v_{j}\right) E_{C}^{A}\left(v_{j}^{*} a v_{i}\right) \phi_{2}\left(v_{i}^{*}\right) \\
& =\sum_{j=1}^{N} \sum_{i=1}^{N} \phi_{1}\left(\left(\operatorname{Index} E_{C}^{A}\right)^{-1}\right) \phi_{1}\left(v_{j}\right) \phi_{2}\left(E_{C}^{A}\left(v_{j}^{*} a v_{i}\right) v_{i}^{*}\right) \\
& =\sum_{j=1}^{N} \phi_{1}\left(\left(\operatorname{Index} E_{C}^{A}\right)^{-1}\right) \phi_{1}\left(v_{j}\right) \phi_{2}\left(v_{j}^{*} a\right)=s \phi_{2}(a) .
\end{aligned}
$$

Taking adjoints gives

$$
s^{*} \phi_{1}(a)=\phi_{2}(a) s^{*}, \quad a \in A
$$

Therefore,

$$
s^{*} s \phi_{2}(a)=s^{*} \phi_{1}(a) s=\phi_{2}(a) s^{*} s, \quad a \in A .
$$

Since $|s| \phi_{2}(a)=\phi_{2}(a)|s|, \phi_{1}(a) u=u \phi_{2}(a)$. Therefore we have that $\phi_{1}=A d(u) \circ \phi_{2}$.

The following proposition shows that sufficiently close intermediate subalgebras with conditional expectations are unitarily equvalent. 
Proposition 3.5. Let $D$ be $a \mathrm{C}^{*}$-algebra and $C$ a $\mathrm{C}^{*}$-subalgebra of $D$ with a common unit and $E_{C}^{D}: D \rightarrow C$ a conditional expectation of finite index. Then, there exists a positive constant $\gamma$ satisfying the following: For any $A, B \in \operatorname{IMS}\left(C, D, E_{C}^{D}\right)$, if $d(A, B)<\gamma$, then there exists a unitary $u \in C^{*}(A, B)$ such that $u A u^{*}=B$. We can choose the unitary in the relative commutant $C^{\prime} \cap D$.

Proof. Let $N$ be the number of a finite basis for $E_{C}^{D}$ in $D_{1}$. Put $\gamma:=(10 N)^{-4}$. By Lemma 3.3, there exists a unital $*$-homomorphism $\psi: A \rightarrow B$ such that $\left.\psi\right|_{C}=i d_{C}$ and

$$
\left\|\left.E_{B}^{D}\right|_{A}-\psi\right\| \leq 8 \sqrt{3} N \sqrt{d(A, B)}<8 \sqrt{3}(100 N)^{-1} .
$$

Since

$$
\left\|\psi-i d_{A}\right\| \leq\left\|\psi-\left.E_{B}^{D}\right|_{A}\right\|+\left\|\left.E_{B}^{D}\right|_{A}-i d_{A}\right\|<8 \sqrt{3}(100 N)^{-1}+2(10 N)^{-4}<\frac{1}{N}
$$

by Lemma 3.2 , there exists a unitary $u \in C^{*}(A, B)$ such that $\psi=A d(u)$ and $\left\|u-1_{D}\right\| \leq$ $\sqrt{2} N\left(8 \sqrt{3}(100 N)^{-1}+2(10 N)^{-4}\right)$ by Lemma 3.4. That is $u A u^{*} \subset B$. For any $b \in B_{1}$, there exists $a \in A_{1}$ such that $\|b-a\| \leq \gamma$ by $d(A, B)<\gamma$. Then

$$
\begin{aligned}
\left\|b-u a u^{*}\right\| & \leq\|b-a\|+\left\|a-u a u^{*}\right\| \\
& \leq \gamma+2\|a\|\left\|u-1_{D}\right\| \\
& \leq(10 N)^{-4}+2 \sqrt{2} N\left(8 \sqrt{3}(100 N)^{-1}+2(10 N)^{-4}\right) \\
& \leq(4 \sqrt{2}+1)(10)^{-4}+16 \sqrt{6}(10)^{-2}<1 ;
\end{aligned}
$$

therefore, $d\left(u A u^{*}, B\right)<1$. By Lemma 2.1, we have that $u A u^{*}=B$.

The following is the main theorem of the paper. Thanks to Izumi's result in [9], we do not need to assume the existence of the conditional expectations onto intermediate $\mathrm{C}^{*}$-subalgebras apriori. We also need the notion minimality of conditional expecations in 12 . Let $D$ be a simple $\mathrm{C}^{*}$-algebra and $C$ a simple $\mathrm{C}^{*}$-subalgebra of $D$ with a common unit and $E_{C}^{D}: D \rightarrow C$ a conditional expectation of finite index. Then there there exists a unique minimal conditional expectation $E_{0}: D \rightarrow C$, that is, Index $E_{0} \leq$ Index $E$ for any conditional expectation $E: D \rightarrow C$ of finite index.

Theorem 3.6. Let $D$ be a simple $\mathrm{C}^{*}$-algebra and $C$ a simple $\mathrm{C}^{*}$-subalgebra of $D$ with a common unit and $E_{C}^{D}: D \rightarrow C$ a conditional expectation of finite index. Then, there exists a positive constant $\gamma$ satisfying the following: For any simple intermediate $\mathrm{C}^{*}$-subalgebras $A$ and $B$ for $C \subset D$, if $d(A, B)<\gamma$, then there exists a unitary $u \in C^{*}(A, B)$ such that $u A u^{*}=B$. We can choose the unitary in the relative commutant $C^{\prime} \cap D$.

Proof. We may assume that $E_{C}^{D}: D \rightarrow C$ is a minimal conditional expectation of finite index by replacing the original conditional expectation by the minimal one, if necesary. Let $A$ be a simple interemediate $\mathrm{C}^{*}$-subalgebra such that $C \subset A \subset D$. Since $E_{C}^{D}$ satisfies Pimsner-Popa inequality, so does the restriction $E_{C}^{A}: A \rightarrow C$. By Corollary 3.4 in Izumi [9], $E_{C}^{A}$ also has a finite basis. Let $\left\{u_{1}, \cdots, u_{N}\right\}$ be a basis for $E_{C}^{A}$. Moreover there exists a conditional expectation $E_{A}^{D}: D \rightarrow A$ of finite index by Proposition 6.1 in Izumi [9]. In fact, we can define $E_{A}^{D}$ by

$$
E_{A}^{D}(x)=\left(\operatorname{Index} E_{C}^{A}\right)^{-1} \sum_{i, j=1}^{N} u_{i} E_{C}^{D}\left(u_{i}^{*} x u_{j}\right) u_{j}^{*}, x \in D .
$$


Replace $E_{C}^{A}$ and $E_{A}^{D}$ by minimal conditional expectations. Then the conposition $E_{C}^{A} \circ E_{A}^{D}$ is also a minimal conditional expectation by Theorem 3 in [12. Since the minimal conditional expectation is unique, $E_{C}^{A} \circ E_{A}^{D}=E_{C}^{D}$. Hence we may assume that the conditional expectation $E_{A}^{D}$ satisfies the compatibility condition. Therefore Proposition 3.5 can be applied.

Remark 3.7. Even if we do not assume that an intermediate $\mathrm{C}^{*}$-subalgebra $A$ is not simple, we can prove a similar fact. But the constant $\gamma$ above depends on the choice of $A$ :

Let $D$ be a simple $\mathrm{C}^{*}$-algebra and $C$ a simple $\mathrm{C}^{*}$-subalgebra of $D$ with a common unit and $E_{C}^{D}: D \rightarrow C$ a conditional expectation of finite index. For any intermediate $\mathrm{C}^{*}$-subalgebras $A$, there exists a positive constant $\gamma$ satisfying the following: For any otheir intermediate $\mathrm{C}^{*}$ subalgebras $B$ for $C \subset D$, if $d(A, B)<\gamma$, then there exists a unitary $u \in C^{*}(A, B)$ such that $u A u^{*}=B$. We can choose the unitary in the relative commutant $C^{\prime} \cap D$. In fact, all we need is that the existance of the conditinal expectation $E_{A}^{D}$ and a finite basis for $E_{C}^{A}$.

In the case of subfactor theory of Jones [10], the lattices of intermediate subfactors with their finiteness were studied in Popa [17, Watatani [20, Teruya-Watatani [18], Longo [15], KhoshkamMashhood [14, Grossman-Jones [8], Grossman-Izumi [7] and Xu [21] for example. In these study, the $\|\cdot\|_{2}$-perturbation technique of von Neumann algebras developed by Christensen [4] are essentially used or motivated.

Since we can choose the implementing unitary $u$ in the relative commutant, we immediately get the following finiteness of the intermediate subalgebras for both simple $\mathrm{C}^{*}$-algebras and factors. A bound of the number is obtained as in Longo [15].

Corollary 3.8. Let $D$ be a simple $\mathrm{C}^{*}$-algebra and $C$ a simple $\mathrm{C}^{*}$-subalgebra of $D$ with a common unit and $E_{C}^{D}: D \rightarrow C$ a conditional expectation of finite index. If the relative commutant $C^{\prime} \cap D$ is trivial, then the number of intermediate $\mathrm{C}^{*}$-subalgebras is finite.

Proof. Since $E_{C}^{D}: D \rightarrow C$ is a conditional expectation of finite index, there exists a dual conditional expectation of finite index $E_{D}: C^{*}\left\langle D, e_{C}\right\rangle \rightarrow D$. Thus, $E_{D} \circ E_{C}^{D}: C^{*}\left\langle D, e_{C}\right\rangle \rightarrow C$ is a conditional expectation of finite index. Since the commutant $C^{\prime} \cap C$ is finite-dimensional, the relative commutant $C^{\prime} \cap C^{*}\left\langle D, e_{C}\right\rangle$ is also finite-dimensional by Proposition 2.7.3 in [19]. Therefore the set

$$
\mathcal{P}:=\left\{p \in C^{\prime} \cap C^{*}\left\langle D, e_{C}\right\rangle \mid p \text { is a projection }\right\}
$$

is a compact Hausdorff space with respect to the operator norm topology. Let $N$ be the number of a finite basis for $E_{C}^{D}$ in $D_{1}$. Let $\varepsilon=\left(2(10 N)^{4}\left\|\operatorname{Index} E_{C}^{D}\right\|\right)^{-1}$. By the compactness, there exists a finite open covering by $\varepsilon$-open balls. For any intermediate $\mathrm{C}^{*}$-subalgebras $A$ and $B$, their Jones projection $e_{A}$ and $e_{B}$ are in $C^{\prime} \cap C^{*}\left\langle D, e_{C}\right\rangle$ and satisfies

$$
d(A, B) \leq\left\|\operatorname{Index} E_{C}^{D}\right\|\left\|e_{A}-e_{B}\right\|
$$

by Lemma 2.6. If two Jones projection $e_{A}$ and $e_{B}$ are in one of these $\varepsilon$-open balls, then $d(A, B)<$ $(10 N)^{-4}$. By Theorem 3.6, there exists a unitary $u$ in $C^{\prime} \cap D$ such that $B=u A u^{*}$. Since $C^{\prime} \cap D=\mathbb{C} I, u$ is a scalar. Therefore $B=A$. This shows that each $\varepsilon$-open ball of the cover contains at most one Jones projection for some intermediate $\mathrm{C}^{*}$-subalgebra. This completes the proof.

Since any subfactor of a type $\mathrm{II}_{1}$ factor has a conditional expectation, we can also apply the same method in this case.

Corollary 3.9. Let $M$ be a type $\mathrm{II}_{1}$ factor and $N$ a subfactor of finite index. If the relative commutant $N^{\prime} \cap M$ is trivial, then the set of intermediate subfactors is a finite set. 


\section{REFERENCES}

[1] D. Bisch, A note on intermediate subfactors, Pacific J. Math., 163 (1994), 201-216.

[2] M. D. Choi and E. Christensen, Completely order isomorphic and close $C^{*}$-algebras need not be $*$-isomorphic, Bull. London Math. Soc., 15 (1983), 604-610.

[3] E. Christensen, Perturbations of type I von Neumann algebras, J. London Math. Soc., 9 (1974/75), 395-405.

[4] E. Christensen, Subalgebras of a finite algebras, Math. Ann., 243 (1979), 17-29.

[5] E. Christensen, Near inclusions of $C^{*}$-algebras, Acta. Math., 144 (1980), 249-265.

[6] E. Christensen, A. M. Sinclair, R. R. Smith, S. A. White and W. Winter, Perturbations of nuclear $C^{*}-$ algebras, Acta. Math., 208 (2012), 93-150.

[7] P. Grossman and M. Izumi, Classification of noncommuting quadrilaterals of factors, Internat. J. Math., 19 (2008), 557-643.

[8] P. Grossman and V. Jones, Intermediate subfactors with no extra structure, J. Amer. Math. Soc., 20 (2007), 219-265.

[9] M. Izumi, Inclusions of simple $C^{*}$-algebras, J. Reine. Angew. Math., 547 (2002), 97-138.

[10] V. Jones, Index for subfactors, Inv. Math. 72 (1983), 1-25.

[11] R. V. Kadison and D. Kastler, Perturbations of von Neumann algebras. I. Stability of type, Amer. J. Math., 94 (1972), 38-54.

[12] S. Kawakami and Y. Watatani, The multiplicativity of the minimal index of simple $C^{*}$-algebras, Proc. Amer. Math. Soc., 123 (1995), 2809-2813.

[13] M. Khoshkam, Perturbations of $C^{*}$-algebras and K-theory, J. Operator Theory, 12 (1984), 89-99.

[14] M. Khoshkam and B. Mashood, On finiteness of the set of intermediate subfactors, Proc. Amer. Math., 132 (2004), 2939-2944.

[15] R. Longo, Conformal subnets and intermediate subfactors, Comm. Math. Phys., 237 (2003), 7-30.

[16] J. Phillips and I. Raeburn, Perturbations of $C^{*}$-algebras II., Proc. London Math. Soc., 43 (1981), 46-72.

[17] S. Popa, Correspondence, preprint.

[18] T. Teruya and Y. Watatani, Lattices of intermediate subfactors for type III factors, Arch. Math., 68 (1997), 454-463.

[19] Y. Watatani, Index for $C^{*}$-subalgebras, Mem. Amer. Math. Soc., 424 (1990).

[20] Y. Watatani, Lattices of intermediate subfactors, J. Func. Anal., 140 (1996), 312-334.

[21] F. Xu, On representing some lattices as lattices of intermediate subfactors of finite index, Adv. Math. 220 (2009), 1317-1356.

(Shoji Ino) Department of Mathematical Sciences, Kyushu University, Motooka, Fukuoka, 8190395, JAPAN.

E-mail address: s-ino@math.kyushu-u.ac.jp

(Yasuo Watatani) Department of Mathematical Sciences, Kyushu University, Motooka, Fukuoka, 819-0395, JAPAN.

E-mail address: watatani@math.kyushu-u.ac.jp 